\title{
Detection of Plasma Circulating Tumor DNA in Ovarian Cancer and Its Clinical Significance
}

\author{
NING NING WANG, MING. YU1 ${ }^{1}$, JIN LU² AND JIN HUA WANG²* \\ Department of Gynecology, ${ }^{1}$ Department of Ultrasonography, Xuzhou Medical College University Affiliated Hospital of \\ Lianyungang \&The First People's Hospital of Lianyungang, Lianyungang, Jiangsu 222000, China; ${ }^{3}$ Department of Gynecologic \\ Oncology, Jiangsu Cancer Hospital \& Jiangsu Institute of Cancer Research \& The Affiliated Cancer Hospital of Nanjing Medical \\ University, Nanjing, Jiangsu 210009, China
}

Ning et al.: Detection of plasma circulating tumor DNA in ovarian cancer

\begin{abstract}
To explore the application value of circulating tumor DNA in the diagnosis of benign and malignant ovarian tumors and its clinical significance in ovarian malignant tumors. A total of 70 patients with ovarian tumors who treated surgery in the first people's hospital of Lianyungang and the affiliated cancer hospital of Nanjing medical university from January 2017 to August 2020 were collected, including 32 cases of benign tumors, 15 cases of cross-sectional tumors, and 23 cases of malignant tumors. Venous blood was collected before the operation, and the expression level of the plasma circulating tumor DNA was detected by Next-Generation Sequencing, and the expression of circulating tumor DNA chromosome copy number variation in benign and malignant ovarian tumors was analyzed. At the same time, the relationship between the preoperative plasma circulating tumor DNA and clinicopathological features such as tumor size, clinical stage, histological grade, pathological type, and related immunohistochemical indexes were analyzed. There was a significant difference in preoperative plasma circulating tumor DNA among patients with benign, cross-sectional, and malignant tumors of the ovary $(p<0.05)$. In terms of the clinical stage, there were 9 cases in the early stage group (I+II), $5(55.56 \%)$ cases were positive for plasma circulating tumor DNA before the operation. In the advanced stage group (III+IV), there were 14 cases, $12(85.71 \%)$ cases were positive for plasma circulating tumor DNA before the operation. In the histological grade of malignant ovarian cancer, there were 6 cases of ovarian low-grade cancer, of which $3(50.0 \%)$ cases were positive for plasma circulating tumor DNA before the operation. There were 17 cases of ovarian high-grade cancer, of which $14(82.35 \%)$ cases were positive for plasma circulating tumor DNA before the operation. Among the pathological types of tumors, there were 16 cases of plasma carcinoma and $14(87.5 \%)$ cases were positive for plasma circulating tumor DNA. There were 7 cases of non-plasma carcinoma and $3(42.85 \%)$ cases were positive for plasma circulating tumor DNA. In terms of tumor size, there were 7 cases with a diameter $<10 \mathrm{~cm}$, of which $3(42.85 \%)$ cases were circulating tumor DNA positive. There were 16 cases of tumor diameter $\geq 10 \mathrm{~cm}$, of which $14(87.5 \%)$ cases were circulating tumor DNA positive. In terms of pathological immunohistochemistry, among the 17 ovarian cancer patients with positive preoperative plasma circulating tumor DNA, there were $14(82.35 \%)$ patients with p53 mutation, and $3(17.65 \%)$ negative patients. There was no significant difference between the detection rate of plasma circulating tumor DNA and the immunohistochemical indexes such as Ki67, P16, estrogen receptor, progesterone receptor, and Wilms tumor protein-1 before the operation. Circulating tumor DNA level has a high predictive value for the disease progression in patients with ovarian cancer, and can be used as a reference index for clinical monitoring of patients' condition and prognosis.
\end{abstract}

Key words: Ovarian cancer,plasma circulatingtumorDNA,clinicalstage,tissuegrade,immunohistochemical indexes

Among the malignant tumors of female reproductive organs, the incidence of ovarian cancer occupies the third place, and its mortality occupies the first place ${ }^{[1]}$. The ovary was located in the deep part of the pelvic cavity, and there were often no obvious clinical symptoms and special discomfort in the early stage of the disease, and there was no sensitive and effective screening method in the early stage of the disease. Although most of the patients come to the hospital when they have clinical symptoms such as abdominal distension, abdominal

*Address for correspondence

E-mail: wangjinhua588@163.com 
pain, massive ascites, and abdominal masses, the patients were often in the advanced stage of the disease. With the metastasis and spread of the tumor, the development of the stage also leads to the difficulty of surgical treatment and poor prognosis. At the same time, more than $50 \%$ of the patients often have recurrence and chemotherapy resistance in the course of treatment, which was a serious threat to the health of the women.

The occurrence and development of ovarian cancer ${ }^{[2]}$ were related to a variety of gene mutations. Therefore, the detection of these mutations in the patients' blood and tumor tissues can not only explore the pathogenesis of the tumor, but also can be used as a specific molecular marker for tumor diagnosis and disease monitoring ${ }^{[3]}$. In recent $y$, with the continuous development of tumor molecular biology and the completion of the Human Genome Project, the minimally invasive and repeatable circulating tumor DNA (ctDNA) detection technology has been widely used in the tumor diagnosis and disease monitoring ${ }^{[4]}$. ctDNA was a DNA fragment released into the blood, which originates from the necrosis and apoptosis of the tumor cells in the primary tumor and its metastatic foci, as well as from the exosome secreted by the tumor cells. ctDNA can not only reflect the tumor $\operatorname{load}^{[5-6]}$, but also contain the biological information originate from the primary tumor ${ }^{[7]}$. At present, there were few studies on ovarian cancer by ctDNA. It has been reported that the ctDNA was detected in peritoneal lavage fluid, ascites, and serum of patients with gynecological malignant tumors such as ovarian cancer, which means that the ctDNA can be used for the detection of ovarian cancer ${ }^{[8,9]}$. However, extreme low concentrations make the detection challenging, particularly at the early stages of tumor development. There were few studies on the ctDNA in ovarian cancer, especially the relationship between ctDNA and clinicopathological features of ovarian cancer $^{[10-12]}$. Therefore, in this study, Next-Generation Sequencing (NGS) was used to detect the plasma ctDNA chromosome copy number variation and its content expression, and to analyze the expression of ctDNA in different ovarian tumors. By detecting the content of ctDNA in patients with different stages of ovarian cancer before the operation, and analyzing the relationship between preoperative plasma ctDNA and cancer antigen 125 (CA125) level, tumor size, Federation of Gynecology and Obstetrics (FIGO) stage, tissue differentiation, postoperative pathological type, $\mathrm{p} 53$, and other clinicopathological features, to further explore the expression of ctDNA in ovarian cancer with different clinicopathological features and its possible influencing factors.

\section{MATERIALS AND METHODS}

\section{Clinical data:}

A total of 70 patients with ovarian tumors were collected in The First People's Hospital of Lianyungang and The Affiliated Cancer Hospital of Nanjing Medical University from January 2017 to August 2020, including 32 cases of benign ovarian tumors, 15 cases of cross-sectional ovarian tumors, and 23 cases of ovarian malignant tumors.

Inclusion criteria: the patients entered the group voluntarily and signed informed consent; patients with good compliance and cooperation of follow-up; the patients without the history of a malignant tumor or history of a malignant tumor and operation in other parts, and did not receive radiotherapy or chemotherapy before the operation; the patients met the criteria of FIGO staging in 2018.

Exclusion criteria: the patients with poor compliance, or unwilling to participate in this study; the patients with hepatitis, colitis, and other long-term chronic inflammation; the patients with serious immune systemic diseases such as systemic lupus erythematosus.

Before the operation, $10 \mathrm{ml}$ of the elbow vein blood was collected from each patient with ovarian cancer for ctDNA detection. All patients treated with surgical treatment, the ovarian benign tumors treated with ovariectomy or adnexectomy, the ovarian crosssectional tumors and malignant tumors treated with staged surgery or ovarian tumor cytoreductive surgery or ovarian cancer exploration. The postoperative pathology was confirmed by two senior pathologists in the department of pathology. P53, Ki67, p16, estrogen receptor (ER), progesterone receptor (PR), Wilms tumor protein-1 (WT-1), and other related immunohistochemical indexes were detected in the cross-sectional and malignant tumors.

All patients with ovarian malignant tumors were staged according to the FIGO 2009 staging criteria, histologically classified and graded according to the World Health Organization (WHO) principles, and treated according to the guidelines for diagnosis and treatment of ovarian cancer.

\section{Extraction and detection of plasma ctDNA:}


Before treatment, $10 \mathrm{ml}$ of elbow vein blood from the patients with ovarian tumor was collected in the Ethylenediaminetetraacetic Acid (EDTA) anticoagulant blood tube, and the tube was gently reversed for 10 times, and the plasma was separated. ctDNA was extracted by HiPure free DNA extraction kit and interrupted to the size of $300 \mathrm{bp}$ by ultrasound. The concentration of free DNA was determined by Qubit dsDNA HS Assay Kit, and the Agilent 2100 fragment was analyzed by High sensitivity DNA reagents kit and High sensitivity DNA chips kit.

\section{ctDNA sequencing and percentage determination:}

NEBNext ${ }^{\circledR}$ Ultra broken DNA Library Prep Kit was used to build the library of DNA. After the segmented DNA was repaired into the blunt-end double-stranded DNA, an adenylate was added at the 3' end as a linker, thus the $\mathrm{T}$ of the 3 ' linker was complementary to the A on the double strand DNA, and the library was amplified by Polymerase chain reaction (PCR). The PCR products were sequenced by NGS.

\section{Observation index:}

The changes of plasma ctDNA content and chromosome copy number in patients with benign, cross-sectional and malignant ovarian tumors were observed and analyzed before the operation. The correlation between the preoperative plasma ctDNA content and preoperative blood CA125 level, tumor size, clinical stage, histological grade, pathological type, p53, and other clinical and pathological factors of ovarian cancer were analyzed.

\section{Statistical analysis:}

SPSS 20.0 software was used for statistical analysis, and chi-square test was used to analyze the counting data. The measurement data were analyzed by t-test and $p<0.05$ means the difference was statistically significant.

\section{RESULTS AND DISCUSSION}

As can be seen from the fig. 1, among the 32 cases of benign ovarian tumors, $2(6.25 \%)$ cases were ctDNA positive, of which $2(6.25 \%)$ cases were ovarian endometrioma, $1(3.13 \%)$ case mature teratoma of the ovary. 15 cases were an ovarian cross-sectional tumor, only $1(6.67 \%)$ case was plasma ctDNA positive. Among 23 cases of ovarian malignant tumors, 17 (73.91\%) cases were ctDNA positive, the difference was statistically significant $(\mathrm{p}<0.05)$.
Among the 23 cases of ovarian malignant tumors, 5 cases were in stage I according to FIGO 2018 stage, and 3 cases were positive for plasma ctDNA. There were 4 cases in stage II and 2 cases were positive in plasma ctDNA. There were 7 cases in stage III, 6 cases were positive in plasma ctDNA and there were 7 cases in stage IV, 6 cases were positive in plasma ctDNA. The tumors were divided into the early stage group (I+II) and the advanced stage group (III+IV). Among them, there were 9 cases in the early group, 5 (55.56\%) cases were positive in plasma ctDNA before the operation. There were 14 cases in the advanced group, 12 (85.71 $\%$ ) cases were positive in plasma ctDNA before the operation, the difference between the two groups was statistically significant ( $<<0.05$, fig. 2).

Among the 23 cases of ovarian malignant tumors, there were 6 cases of ovarian low-grade cancer, of which $3(50.0 \%)$ cases were positive for plasma ctDNA. There were 17 cases of ovarian high-grade cancer, of which

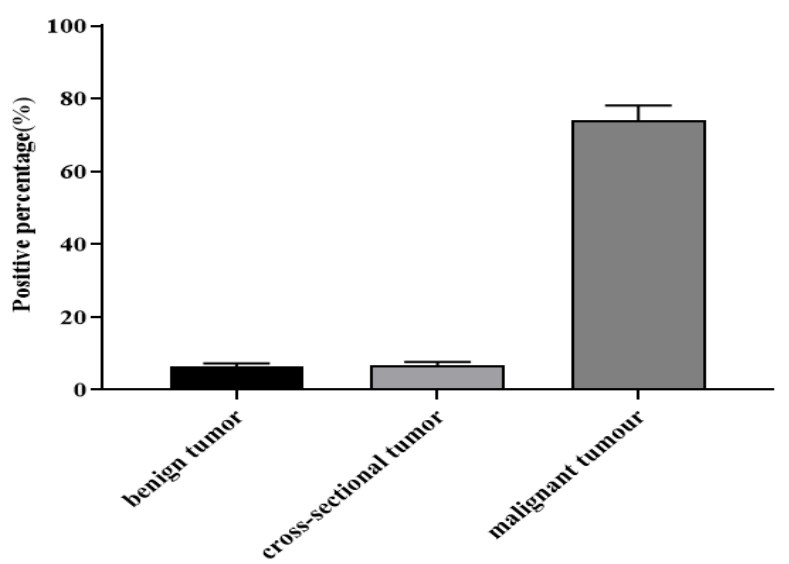

Fig. 1: Preoperative plasma ctDNA expression in patients with benign, cross-sectional, and malignant ovarian tumors

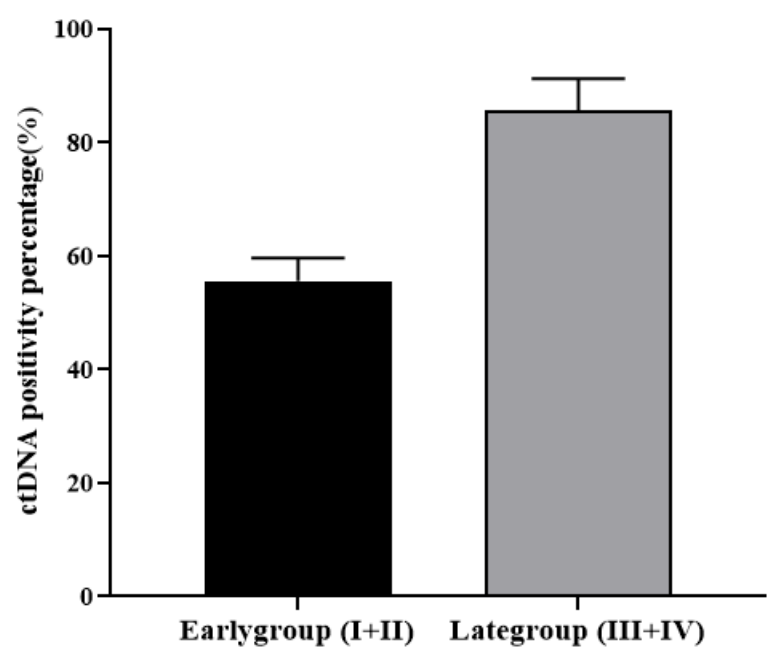

Fig. 2: Relationship between the preoperative plasma ctDNA and clinical stage of ovarian malignant tumor 
$14(82.35 \%)$ cases were positive for plasma ctDNA. The difference was statistically significant $(\mathrm{p}<0.05$, fig. 3).

A total of 23 cases of ovarian malignant tumors were divided into two groups, plasma carcinoma group and non-plasma carcinoma group. Among 16 cases of plasma carcinoma, $14(87.5 \%)$ cases were ctDNA positive. Among the 7 cases of non-plasma carcinoma, $3(42.86 \%)$ cases were ctDNA positive, the difference was statistically significant ( $<<0.05$, fig. 4 ).

A total of 23 cases of ovarian malignant tumors were divided into 2 groups according to tumor size. The tumor diameter of 7 patients was less than $10 \mathrm{~cm}$, of which $3(42.86 \%)$ cases were ctDNA positive. The tumor diameter of 16 patients was $\geq 10 \mathrm{~cm}$, of which $14(87.5 \%)$ cases were ctDNA positive. The difference was statistically significant $(p<0.05)$. With the increase of tumor diameter, the positive rate of ctDNA increased significantly. There was a correlation between the detection rate of plasma ctDNA and tumor size before

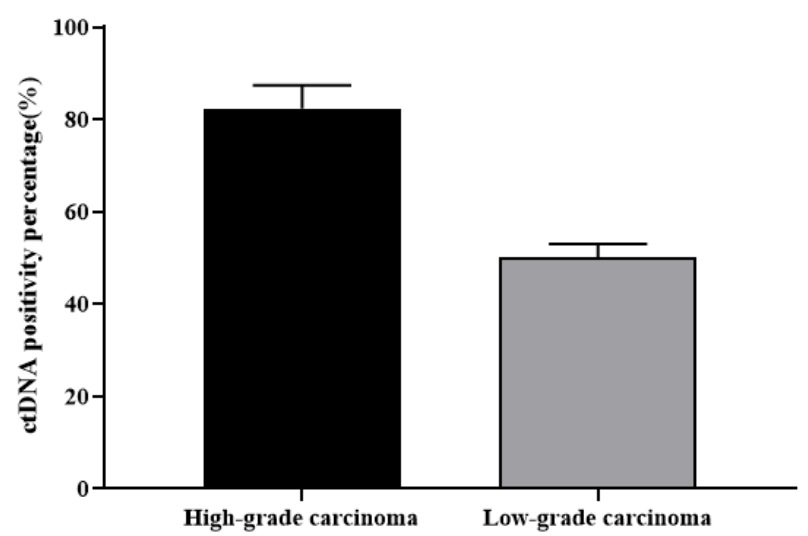

Fig. 3: Relationship between the preoperative plasma ctDNA and histological grade of ovarian malignant tumor

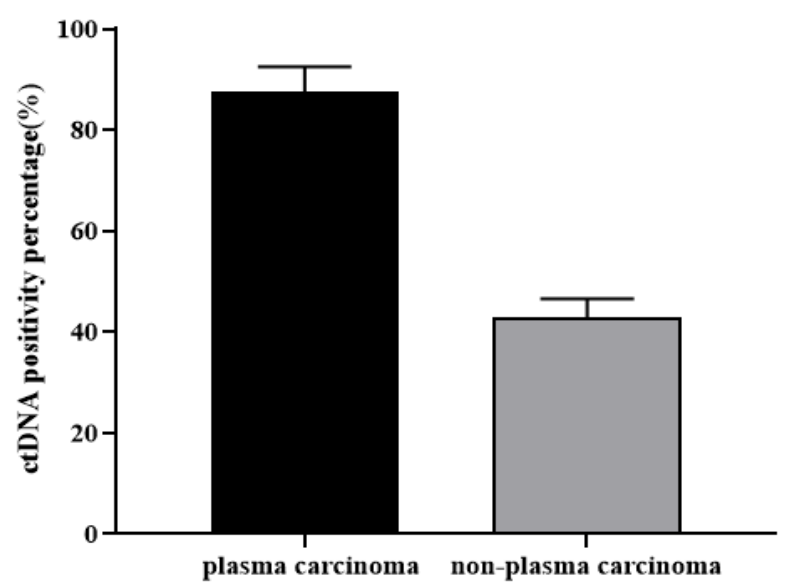

Fig. 4: Relationship between preoperative plasma ctDNA and pathological types of ovarian malignant tumors
P53, Ki67, P16, ER, PR, WT-1, and other immunohistochemical indexes were detected in the 23 ovarian cancer patients after operation. The results in Table 1 showed that there was a correlation between preoperative plasma ctDNA detection rate and p53 mutation. Among the 23 cases of ovarian cancer, 17 cases were preoperative blood ctDNA positive patients, $14(82.35 \%)$ cases of immunohistochemical p53 mutation patients, and 6 cases of preoperative blood ctDNA negative patients, tissue immunohistochemistry detected p53 mutation patients $3(50 \%)$ cases, the agreement reached $73.91 \%$. The difference was statistically significant $(p<0.05)$. However, there was no significant difference between the detection rate of plasma ctDNA and the immunohistochemical indexes such as Ki67, p16, ER, PR, and WT-1 before the operation.

Ovarian cancer was the third most common gynecological malignant tumor in the world, and it was the main cause of death of patients with gynecological malignant tumor ${ }^{[13]}$. Epithelial ovarian cancer was the main type of ovarian malignant tumor, with histology and molecular heterogeneity, which was a highly invasive malignant tumor. In the past $30 \mathrm{y}$, little progress has been made in the standard chemotherapy and overall survival (OS) for ovarian cancer ${ }^{[14]}$. The main treatment was surgery to remove the tumor lesions and to achieve the tumor cell reduction as far as possible, and combined with postoperative chemotherapy (paclitaxel+platinum $)^{[15-17]}$. However, chemotherapy resistance and disease recurrence were observed in more than half of the cases ${ }^{[18,19]}$.

In recent $y$, with the continuous development of NGS

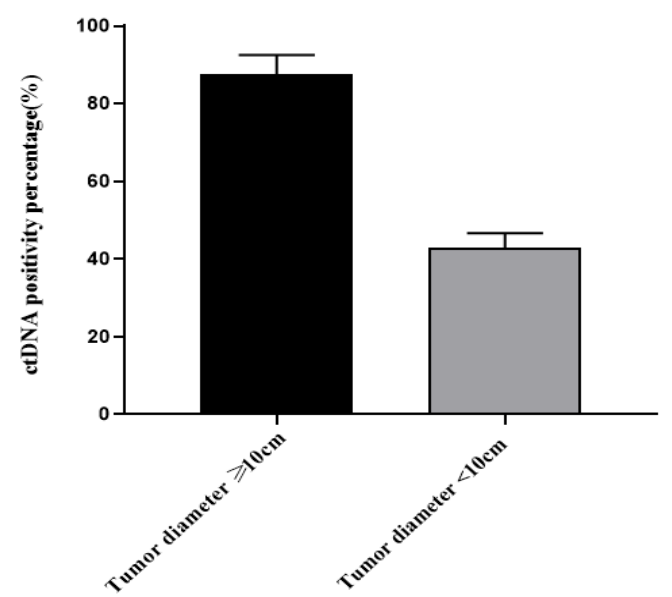

Fig. 5: The correlation between preoperative plasma ctDNA and tumor size in patients with ovarian malignant tumor Special Issue 7, 2020 


\begin{tabular}{|c|c|c|c|c|c|}
\hline \multicolumn{3}{|c|}{$\begin{array}{l}\text { TABLE 1: } \\
\text { PREOPERATIVE } \\
\text { PATHOLOGICAL } \\
\text { INDEXES }\end{array}$} & \multicolumn{3}{|c|}{$\begin{array}{c}\text { CORRELATION BETWEEN } \\
\text { PLASMA ctDNA AND } \\
\text { IMMUNOHISTOCHEMICAL }\end{array}$} \\
\hline & & ctDI & $\begin{array}{l}\text { NA negative } \\
(n=6)\end{array}$ & $\begin{array}{c}\text { ctDNA positive } \\
(n=17)\end{array}$ & $p$ Value \\
\hline \multirow[t]{2}{*}{ P53 } & Negative & & 3 & 3 & \multirow{2}{*}{0.017} \\
\hline & Positive & & 3 & 14 & \\
\hline \multirow[t]{2}{*}{ Ki67 } & Negative & & 4 & 8 & \multirow{2}{*}{0.741} \\
\hline & Positive & & 2 & 9 & \\
\hline \multirow[t]{2}{*}{ ER } & Negative & & 3 & 9 & \multirow[b]{2}{*}{0.792} \\
\hline & Positive & & 3 & 8 & \\
\hline \multirow[t]{2}{*}{ PR } & Negative & & 2 & 8 & \multirow[b]{2}{*}{0.821} \\
\hline & Positive & & 4 & 9 & \\
\hline \multirow[t]{2}{*}{ WT-1 } & Negative & & 3 & 8 & \multirow[b]{2}{*}{0.733} \\
\hline & Positive & & 3 & 9 & \\
\hline
\end{tabular}

technology, ctDNA has been widely used in the tumor diagnosis, treatment, and prognosis detection ${ }^{[17]}$. It has been reported that the ctDNA detection can distinguish the early lung cancer and benign lung tumors successfully in the case of negative tumor markers and imaging findings of small lesions. Some scholars have found that the ctDNA can detect the ovarian cancer patients with false negative CT in 22 ovarian cancer patients, and the results of ctDNA were related to the prognosis of patients ${ }^{[20]}$. This study found that among the 23 cases of ovarian malignant tumors, there were 18 patients with preoperative blood CA125>35 $\mu / \mathrm{ml}$, with a positive rate of $78.26 \%$. And $17(73.91 \%)$ patients with positive plasma ctDNA before the operation. The difference was not statistically significant. Preoperative plasma ctDNA, like CA125, can be used as a new "fluid biopsy" method in the diagnosis of ovarian malignant tumors.

Some studies have found that the detection rate of plasma ctDNA varies with the type and stage of malignant tumor ${ }^{[20]}$. The results of Tian et al. ${ }^{[21]}$ showed that the positive rate of plasma ctDNA in patients with lung cancer was $90 \%$, and the detection rate of patients with early lung cancer was $85 \%$. In the breast cancer, the detection rate of plasma ctDNA in the early patients was about $50-70 \%{ }^{[22]}$. The study of Fader et al..$^{[23]}$ confirmed that the detection rate of plasma ctDNA in patients with stage I-II ovarian cancer was $63.6 \%$. Our study had found that among the 23 cases of ovarian malignant tumors, 9 cases were early ovarian cancer, and 5 cases $(55.56 \%)$ were ctDNA positive before the operation, which was lower than the detection rate of the early lung cancer and early breast cancer reported in the above literature, and lower than the ctDNA detection rate of early ovarian cancer reported by Fader et al.
Of the 14 patients with advanced ovarian cancer, 12 $(85.71 \%)$ were plasma ctDNA positive before the operation, which was similar to that reported in the literature. It was significantly higher than that of the early ovarian cancer, and the difference was statistically significant. Ovarian cancer was mainly abdominal colonization and lymphatic metastasis, and the blood metastasis occurs only in the advanced stage. Thus the preoperative detection rate of plasma ctDNA in the early ovarian cancer was low, while the preoperative detection rate of plasma ctDNA in the advanced ovarian cancer was significantly higher. It was suggested that the preoperative plasma ctDNA has a certain application value in the diagnosis of advanced ovarian cancer.

Whether there was a correlation between the preoperative plasma ctDNA and the size of ovarian malignant tumor, there were few reports at home and abroad ${ }^{[24]}$. Study has found that the number of circulating tumor cells in the blood of patients with tumor diameter $\geq 10 \mathrm{~cm}$ was significantly higher than that of patients with tumor diameter $<10 \mathrm{~cm}$. According to the above research, we take the tumor size $10 \mathrm{~cm}$ as the boundary. Among the 23 cases of ovarian malignant tumors, there were 7 cases with tumors diameter $<10 \mathrm{~cm}$, including 3 cases with ctDNA positive. The preoperative blood ctDNA positive rate of cases was $42.86 \%$. There were 16 cases with tumors $\geq 10 \mathrm{~cm}$, including 14 cases with ctDNA positive. The positive rate of blood ctDNA is $87.5 \%$. These results showed that with the increase of tumor volume, the tumor load increases, the amount of ctDNA released into plasma increases significantly, and the positive rate of plasma ctDNA increases significantly before the operation. There was a correlation between the detection rate of plasma ctDNA before the operation and the size of tumor.

This study showed that among 23 cases of ovarian malignant tumors, 6 cases were low-grade ovarian cancer, of which 3 cases were positive for ctDNA preoperative blood, which accounted for $13.04 \%$; 17 cases were high-grade ovarian cancer, of which 14 cases were preoperative blood. ctDNA positive, preoperative blood ctDNA positive accounted for 60.87 $\%$, The difference was statistically significant, $\mathrm{p}<0.05$. The detection rate of the preoperative plasma ctDNA in patients with high-grade ovarian cancer was higher. The possible mechanism was that with the decrease of tumor differentiation and the increase of malignant degree, the invasiveness of tumor ${ }^{[25]}$ increases obviously. And the probability of ctDNA release into blood increases, suggesting that the content of the preoperative plasma 
ctDNA may be related to the differentiation degree of the ovarian malignant tumor.

Some studies have found that the detection rate of plasma ctDNA varies with the type and stage of malignant tumor ${ }^{[26]}$. In this study, 23 cases of ovarian cancer were divided into two groups, plasma carcinoma and non-plasma carcinoma. The results showed that the detection rate of ctDNA in plasma ovarian carcinoma was significantly higher than that in non-plasma ovarian carcinoma. The sensitivity of ctDNA in plasma ovarian carcinoma was significantly better than that of non-plasma ovarian carcinoma. In 16 cases of plasma ovarian carcinoma, the positive rate of ctDNA in highgrade plasma ovarian carcinoma was significantly higher than that in low-grade plasma carcinoma. It was more sensitive to detect preoperative plasma ctDNA in high-grade plasma ovarian carcinoma based on NGS.

P53 gene was the most widely and deeply studied tumor suppressor gene at present, and more than $50 \%$ of tumors were related to this gene. P53 gene was located on human chromosome $17 \mathrm{p} 13.1$, with a total length of about $20 \mathrm{~kb}$, which was a negative regulator in the cell growth cycle. Lee et al. ${ }^{[27]}$ detected $\mathrm{p} 53$ gene mutations in the preoperative plasma ctDNA of ovarian cancer patients, and found that $95 \%$ of the patients had mutations. Yong-Man et al. ${ }^{[28]}$ directly sequenced the tumor tissues of 61 high-grade serous ovarian cancer (HGSC) patients and confirmed p53 mutations in 41 cases $(67.2 \%)$. Somatic mutations in all patient-specific tissues could be detected in plasma ctDNA, with a coincidence rate of $100 \%$. The level of p53 mutation ctDNA has a significant prognostic value 3 mo after treatment compared with CA125, suggesting that p53 mutation ctDNA was expected to become a biomarker of HGSC treatment response monitoring. This study showed that the detection rate of ctDNA in the preoperative plasma of ovarian cancer was closely related to p53 mutation, especially in high-grade plasma carcinoma. Among the 23 cases of ovarian malignant tumors, 14/17 ctDNA positive patients had p53 gene mutation, with a mutation rate of $82.35 \%$. The difference was statistically significant, $\mathrm{p}<0.05$. Our results were similar to those reported in the literature.

To sum up, the level of ctDNA has a high predictive value for the disease progression of patients with ovarian cancer, and can be used as a reference index for clinical monitoring of patients' condition and prognosis.

\section{Acknowledgement:}

This work was supported by Jiangsu Provincial
Research project on Maternal and Child Health (No. F201706). NING NING. WANG and MING. YU contributed equally to this work.

\section{Declaration of interest:}

The authors report no conflicts of interest.

\section{REFERENCES}

1. Torre LA, Bray F, Siegel RL, Ferlay J, Lortet-Tieulent J, Jemal A. Global cancer statistics, 2012 CA Cancer J Clin 2015;65:87108.

2. Miller KD, Goding Sauer A, Ortiz AP, Fedewa SA, Pinheiro PS, Tortolero-Luna G, et al. Cancer statistics for hispanics/ latinos, 2018. CA Cancer J Clin 2018;68:425-45.

3. Howlader N, Noone AM, Krapcho M. SEER Cancer Statistics Review,1975-2014, based on November 2016 SEER data submission. MD National Cancer Institute 2017.

4. Chen W, Zheng R, Baade PD, Zhang S, Zeng H, Bray F, et al. Cancer statistics in China, 2015. CA Cancer J Clin 2016;66:115-32.

5. Patch AM, Christie EL, Etemadmoghadam D, Garsed DW, George J, Fereday S, et al. Whole-genome characterization of chemoresistant ovarian cancer. Nature 2015;521:489-94.

6. Zhu CS, Huang WY, Pinsky PF, Berg CD, Sherman M, Kelly JY, et al. The Prostate, Lung, Colorectal and Ovarian Cancer (PLCO) screening trial pathology tissue resource. Cancer Epidemiol Prev Biomarkers 2016;25:1635-42.

7. Gold B, Cankovic M, Furtado LV, Meier F, Gocke CD. Do circulating tumor cells exosomes, and circulating tumor nucleic acids have clinical utility? A report of the association for molecular Pathology. J Mol Diagn 2015;17:209-24.

8. Perkins G, Yap TA, Pope L, Cassidy AM, Dukes JP, Riisnaes R, et al. Multi-purpose utility of circulating plasma DNA testing in patients with advanced cancers. PloS one 2012;7:e47020.

9. Esposito A, Bardelli A, Criscitiello C, Colombo N, Gelao L, Fumagalli L, et al. Monitoring tumor-derived cell-free DNA in patients with solid tumors: clinical perspectives and research opportunities. Cancer Treat Rev 2014;40:648-55.

10. Madic J, Kiialainen A, Bidard FC, Birzele F, Ramey G, Leroy $\mathrm{Q}$, et al. Circulating tumor DNA and circulating tumor cells in metastatic triple negative breast cancer patients. Int J Cancer 2015;136:2158-65.

11. Giannopoulou L, Kasimir-Bauer S, Lianidou ES. Liquid biopsy in ovarian cancer: recent advances on circulating tumor cells and circulating tumor DNA. Clin Chem Lab Med 2018;56:186-97.

12. Swisher EM, Wollan M, Mahtani SM, Willner JB, Garcia R, Goff BA, et al. Tumor-specific p53 sequences in blood and peritoneal fluid of women with epithelial ovarian cancer. Am J Obstet Gynecol 2005;193:662-7.

13. Forshew T, Murtaza M, Parkinson C, Gale D, Tsui DW, Kaper $\mathrm{F}$, et al. Noninvasive identification and monitoring of cancer mutations by targeted deep sequencing of plasma DNA. Sci Transl Med 2012;4:136ra68.

14. Siegel RL, Miller KD, Jemal A. Cancer statistics, 2015. CA Cancer J Clin 2015;65:5-29.

15. Du Bois A, Pfisterer J. Future options for first-line therapy of advanced ovarian cancer. Int J Gynecol Cancer 2005;15:42-50.

16. Du Bois A, Reuss A, Pujade-Lauraine E, Harter P, Ray-Coquard I, Pfisterer J. Role of surgical outcome as prognostic factor in 
advanced epithelial ovarian cancer: a combined exploratory analysis of 3 prospectively randomized phase 3 multicenter trials: by the Arbeitsgemeinschaft Gynaekologische Onkologie Studiengruppe Ovarialkarzinom (AGO-OVAR) and the Grouped'Investigateurs Nationaux Pour les Etudes des Cancers de 1'Ovaire (GINECO). Cancer 2009;115:1234-44.

17. Zhu CS, Pinsky PF, Cramer DW, Ransohoff DF, Hartge P, Pfeiffer RM, et al. A framework for evaluating biomarkers for early detection: validation of biomarker panels for ovarian cancer. Cancer Prev Res 2011;4:375-83.

18. Kirwan A, Utratna M, O’Dwyer ME, Joshi L, Kilcoyne M. Glycosylation-based serum biomarkers for cancer diagnostics and prognostics. Biomed Res Int 2015;2015:490531.

19. Chae YK, Davis AA, Carneiro BA, Chandra S, Mohindra N, Kalyan A, et al. Concordance between genomic alterations assessed by next-generation sequencing in tumor tissue or circulating cell-free DNA. Oncotarget 2016;7:65364-73.

20. Jovelet C, Ileana E, Le Deley MC, Motté N, Rosellini S, Romero A, et al. Circulating cell-free tumor DNA analysis of 50 genes by next-generation sequencing in the prospective MOSCATO trial. Clin Cancer Res 2016;22:2960-8.

21. Tian G, Li X, Xie Y, Xu F, Yu D, Cao F, et al. The early diagnosis in lung cancer by the detection of circulating tumor DNA. BioRxiv 2017:189340.

22. Giannopoulou L, Mastoraki S, Buderath P, Strati A, Pavlakis K, Kasimir-Bauer S, et al. ESR1 methylation in primary tumors and paired circulating tumor DNA of patients with high-grade serous ovarian cancer. Gynecol Oncol 2018;150:355-60.

23. Fader AN, Wang Y, Papadapoulos N, Stone RL, Wang TL, Tanner EJ, et al. Detection of tumor-derived DNA with combination Pap smear and plasma testing in women with primary ovarian cancer: A potential screening test on the horizon?. Gynecol Oncol 2017;145:24-5.

24. Canzoniero JV, Park BH. Use of cell free DNA in breast oncology. Biochim Biophys Acta 2016;1865:266-74.

25. Ocana A, Díez-González L, García-Olmo DC, Templeton AJ, Vera-Badillo F, Escribano MJ, et al. Circulating DNA and survival in solid tumors. Cancer Epidemiol Prev Biomarkers 2016;25:399-406.

26. Ai B, Liu H, Huang Y, Peng P. Circulating cell-free DNA as a prognostic and predictive biomarker in non-small cell lung cancer. Oncotarget 2016;7:44583-95.

27. Lee HY, Lee SW, Na GH, Lee SE, Kang DW, Kim YM. Abstract B04: early detection of ovarian cancer recurrence using p53-mutated circulating tumor DNA as non-invasive biomarkers. Clin Cancer Res 2016;22:B4.

28. Kim YM, Lee SW, Lee YJ, Lee YH, Lee JE, Choi EK. Prospective study of the efficacy and utility of TP53 mutations in circulating tumor DNA as a non-invasive biomarker of treatment response monitoring in patients with high-grade serous ovarian carcinoma. Gynecol Oncol 2019;30:e32.

This is an open access article distributed under the terms of the Creative Commons Attribution-NonCommercial-ShareAlike 3.0 License, which allows others to remix, tweak, and build upon the work non-commercially, as long as the author is credited and the new creations are licensed under the identical terms

This article was originally published in a special issue,
"Biomedical Research in Clinical and Preclinical
$\begin{aligned} & \text { Pharmaceutics" Indian J Pharm Sci 2020:82(5)Spl } \\ & \text { issue7;145-151 }\end{aligned}$

